\title{
The use of computer-aided teaching software in the teaching of basketball tactics
}

\author{
Yanjun Chang \\ Authors Name/s per 1st Affiliation (Author) \\ Institute of Physical Education of Langfang Teacher's College; \\ Langfang, Beijing \\ e-mail:changjinqi@yahoo.com.cn
}

\begin{abstract}
The development of computer-aided technology provides the material basis for the construction of the integration of learning theory and basketball tactics course. But computer-aided technology itself does not naturally promote teaching, how it reasonable design, the use of computer-aided technology can fully reflect the of basketball tactical movement skills learning principles? Embodies the law of basketball tactics and skills in learning how to make the whole process of teaching and characteristics, in order to achieve the purpose of the optimization of teaching? To solve this problem, basketball Tactical Teaching must be systematic design. (Abstract)
\end{abstract}

Keywords-component; Computer-aided technologies; Constructivism; Basketball; Tactical Teaching (key words)

\section{INTRODUCTION (HEADING 1)}

Into the 21st century, with the deepening of the reform of teaching and modern educational technology the continuous development of, computer technology with it's efficient, fast, and has gradually been applied to modern teaching aspects. Computer-aided instruction can simulate or interactive control some realistic image video, and able to provide the best teaching and learning environment for teachers. Longterm computer-aided teaching practice has proved that the rational use of computer-aided teaching will help improve students' learning enthusiasm and initiative, abstract or difficult performance of continuous things specific, static, help students quickly understand and master, maximizing develop learning efficiency and classroom teaching. Currently, various institutions on the basis of active research and development and use of a variety of computer-aided teaching software. Computer Aided Instruction as a favorable means of teaching reform in the teaching of basketball tactics also is actively promoted.

The Basketball Teaching is a strong imitative movement, the traditional teachings general teacher lectures and demonstrations, but because of the teaching requirements during exercise basketball intuitive, the specific timing, especially with regard to the tactical choice of the form of tactical teaching capture, etc., rely solely on teachers, and lectures, draw a schematic diagram of a spot anyway, to be comprehensive, very difficult image to explain. Because of tactical demonstration is people (including the offensive and defensive players) synchronous interactive process, teachers need more than one position demonstration, the need to make clear to start timing. The entire process of tactical, single explanation and demonstration by teachers is more abstract, and difficult to form a global tactical awareness. Laborious and time-consuming, and therefore the teaching of basketball tactics need to change the status quo, while the computer-aided basketball teaching video presentation, tactics animation, the time to explain, interactive operation integrated treatment of a variety of techniques, not only can virtual building a basketball court, basketball, athletes and other material, simulation of basketball tactics can simulate real background, analysis of basketball tactics engine timing, tactical routes, and inspire students to technological innovation, so that students easily formed tactical awareness and movement representation, to improve the efficiency and effectiveness of teachers basketball Tactical Teaching.

\section{FEATURE.}

First, the use of computer-aided teaching software illustrated, can the characteristics of the virtual scene, create the image, interactive, dynamic and static context for classroom teaching, can promote students' eyes, ears, hands, brain and other senses stimulated at the same time, induced and maintained interest in learning, and enhance the initiative to participate in teaching. Followed by the function of the computer-aided simulation demonstrates the teaching content becomes interesting abstract theory, as well as difficult to solve the problems of language and teaching aids demonstration visualize intuitively attractive.[1] Fast and convenient multimedia-specific, beyond time and space superiority can narrow the distance of space, and to shorten the time-consuming process. Traditional teaching methods, teachers again using chalk on a blackboard teaching, timeconsuming and labor-intensive. And the use of multimediaassisted teaching, only need to scan this information into the computer and the appropriate editing adjustments, handy in the classroom can be in accordance with the teaching program sound, like maps, text presented is clear and standardized. This saves a classroom blackboard, to avoid the distraction of students, increased classroom capacity to transmit information. Class, with just a click of a mouse, teaching content will individually display greatly accelerate the pace of teaching. Teaching content nearly half of the more than usual, but the students within the stipulated time easily learn more knowledge. 


\section{DOMESTIC BASKETBALL COMPUTER-AIDED TEACHING THEORY RESEARCH}

Most of the articles are limited to theoretical principles, experimental training but less specific development technology, the principle. Mattress group on "computer applications Digest" published the "Photoshop round to your basketball dreams" use Photoshop basketball cartography, Peng three eagles on the "Hubei Sports Science and Technology" published the "basketball tactical foundation with the multimedia courseware for the development and application of" use of Authorware Wo POWERPOINT software technical action shot of a basketball jump shot timing "Flash technology, Ma" Neijiang technology ", using Power point technology. In addition, some articles Imagination multimedia how to use basketball as JILIN INSTITUTE Sports Adult Education ", 2004" published "virtual reality technology aided teaching of basketball" proposed the use of virtual technology, but in fact 2010years of 3D cinema a late start, not many used in other areas, 2010, Qi Lin Shandong Institute of Physical Education "'" college basketball teaching multimedia network courseware development and application of "Pose technology, which is action figures software would like to use this soft make the pieces character animation is very difficult, the authors propose the idea, but in practice it is too difficult. Can be seen through the above literature, computer-aided technology in basketball training, teaching, practical exercise, psychological testing and other aspects of the theory has to have some basis, but most of the research has focused its research significance, its real design research is still quite small, its research in this field is quite weak, not yet seen by the public, the widely accepted use of achievements. Although some domestic scholar's sports community basketball computer-aided instructional design has started to pay attention, but the problem is still relatively shallow, confined mainly to the practical application of comparative effectiveness level, that did not reach the level of theory, there are no substantive results.

\section{ADVANTAGE}

Before you begin to format your paper, first write and save the content as a separate text file. Keep your text and graphic files separate until after the text has been formatted and styled. Do not use hard tabs, and limit use of hard returns to only one return at the end of a paragraph. Do not add any kind of pagination anywhere in the paper. Do not number text heads-the template will do that for you.

First, to improve the traditional teaching methods, the traditional teaching difficulties solved. An important course of basketball as a school sports content loved by the students. Over the years, the teaching of basketball generally just through the teacher's explanations and personal demonstration. For such a technical and tactical flexibility in terms of the collective project, traditional conventional teaching alone is difficult to achieve better teaching results in the use of certain limitations. Basketball tactics explain the scene by explaining Let students imagine the tactical use, many people with the opportunity to grasp the use of the action, almost difficult to accomplish. The same is also difficult to achieve through demonstration, because students difficult tactical formation the overall continuous awareness, not to the use of tactical fighter, with the space to form a representation of consciousness, which in turn is difficult to understand and use, and not produce the desired effect. And the use of computer-aided multimedia software, sound, pictures, video, animation, and computer-aided software slow motion replay, annotation, suggestive language can be intuitive, image, dynamic presentation, so that not only can help to cover the body language deficiencies and oral presentations, to help students fully understand the tactical principle, can also help students form a tactical awareness, improve teaching efficiency and effectiveness, and to make up for the deficiencies of the traditional teaching methods.

Followed by computer-aided technology gradually improve tactical awareness. Basketball tactical awareness is in the process of basketball player's brain actively thinking of a process, the formation of such thinking is to deepen the understanding of the players on the basketball regular as well as individual basketball skills ability in basketball practice, a reflection of the brain for knowledge. This reflects the ability to build a basketball understand the role of psychological and physiological, refine and reflect a long process. Basketball consciousness mainly includes observation skills, analytical skills, rapid response capability, tactical thinking, adaptability, self-control situation. The process is divided into several levels of basketball consciousness: To observe the perception process, the process of critical thinking, action-reaction process, feedback process. These processes affect the formation of the basketball tactical awareness. Basketball awareness generally obtained by basketball player from learning and real-world experience, and the slow accumulation of knowledge in some other way. [2] where the use of computer-aided technology, demonstrated through the video in the form of image movement routes, guide each position player to perceived competition scene by observing the movement routes, the tactical coordination analysis to determine the respective positions, and select the appropriate time to make the options for action. Consolidate tactical thinking virtual some real-world scenarios to guide students to make the analysis and judgment, to students, the ability to judge. Some network basketball games, players can set up their own team's offensive and defensive strategy, you can select the position in any way offensive or defensive, and then control the game, this control ability is also a need to gradually build can be formed.[3]

Again to strengthen the students' memory upgrade. Knowledge learned in the study and practice of the movement, the students understanding of the feedback to the brain through the sense, and has ordered the conduct of visual, auditory, tactile, judgment and memory. Finally, organized screening useful information to form a final memory of thinking, mutual coordination of a variety of senses can memorize things better. The experiments show that a variety of senses at the same time participate in the class, the students are indeed able to better understand and memory problems, and greatly improve the efficiency of learning in the limited class time. Computer-aided techniques 
that combine images, text, animation, sound more sensory required, many ways to stimulate the human brain memory cells, so as to achieve a higher level of learning efficiency.

\section{INSTRUCTIONAL DESIGN}

After the text edit has been completed, the paper is ready for the template. Duplicate the template file by using the Save As command, and use the naming convention prescribed by your conference for the name of your paper. In this newly created file, highlight all of the contents and import your prepared text file. You are now ready to style your paper.

Basketball tactical computer aided instruction software framework. Based on confrontation basketball features, usually divided into two major systems of offensive and defensive basketball skills.

\section{A. Attacks}

\section{1) Quick Attack}

a) Dribble advancing trio Three biographies

b) Three-wire fast break off the bit

c) Parallel three-two.The vertical bit hit two

2) offensive zone defense

a) Attack 2-1-2 defense

b) 1-3-1 down dual center empty cut

c) 1-2-2 double center bottom line to cover

3) The offensive half marking single post attack method

a) 1-3-1 bottom line mobile

b) 2-1-2 offensive attack halftime marking single center attack method

c) 2-1-2 mass cut cover

\section{B. Defensive tactics}

1) anti-fast break the blocking of the card blocking the end of the fast break

2) Zonal Defense

3) On Half Court Person to Person Defense and Team Work

4) All Court Press

5) mixed defend

Basketball tactical computer aided instruction software system design. Design software framework, requiring simple, crisp, convenient and practical, does not require too much modification, saving as much as possible to stay out of the main content presentation available space, the size of the fight for the resolution of each icon, consider the interface to enlarge when icons and pictures for the future may be the increase of new features (such as: menus, labels, buttons) leave a spare position. Teaching content of computer-aided software, how scientific and rational design of software framework, which would require the use and design of software structures have a certain understanding of the people, to be able to be in the process of designing the entire color decoration effective combinations where the place the main menu, there is a page content using conventional visual effect, consider the use of user comments, so that the overall framework for crystal clear at a glance.

Basketball tactical computer aided instruction software overall production. The software developed specific guiding ideology and scripts, teaching objectives, teaching content, teaching strategies and activities, as well as the function module of the overall design thinking. Audio fast split conversion tools, Photoshop and other multimedia authoring software, text, video, images and other material processing, flash8.0 overall production of the basketball tactics computer-aided teaching software. And the entire software creative thinking and creative composition final perfection. [5] of the entire software from start to finish, each interface, each feature comprehensive "scan" to complete the system synthesis. Basketball tactical computer aided instruction software is to the computer as a teaching core media, through over integrated processing of sounds, images, text, graphics, audio, video, animation and other media information, the multi by Flash8.0 software overlay technology to build virtual plane basketball court, basketball, athletes and other material, and import background music, game sound effects and commentary sound basketball tactics simulation analysis of the route, the timing and the advantages and disadvantages of basketball tactics and tactical innovation, the establishment of a large number of logical connections between basketball tactics teaching information, integrated interactive teaching system . The multimedia CAI technology to the development of basketball tactics teaching human-computer interaction software so students basketball skills and tactics with the initial establishment of perceptual knowledge, to improve the effect of the basketball training teachers on the teaching of technical and tactical.

\section{REFERENCES}

[1] Huang Fu and India. Basketball Tactical Teaching multimedia animation techniques in [D]. Guangxi Normal University, 2006.

[2] Dong Deping. Basketball tactical foundation with the web-based multimedia CAI courseware development and teaching applied research [D]. North Beijing Sports University, 2005.

[3] Liang Xiuping. The application of multimedia technology in the basketball teaching [J]. Liaoning Higher Vocational Technical Institute, 2002 (2) :75-76.

[4] Xie iron rabbit editor. Basketball Tactics Teaching methods and steps [M]. Beijing: Beijing Sports University Press, 2010.

[5] Mattress group. Photoshop regiment your basketball dreams [J]. The computer applications Wencui the, 2002 (3) :49-50. 